\title{
Studying the Interrelationship amongst various Challenges for Adoption of Technologies in Indian Health Care Ecosystem
}

\author{
Arnav Jain \\ Techture Structures Private \\ Limited, Indore, India
}

\author{
Remica Aggarwal \\ School of Business, University \\ of Petroleum \& Energy Studies, \\ Dehradun, India
}

\author{
Veena Aggarwal \\ Recventures Education \\ Services Private Limited \\ Delhi, India
}

\begin{abstract}
Health care is one of the fastest growing sectors in India and Indian government has aggressive plans to develop India further into global health care hub. It presents a great opportunity for the growth of the entire health industry medical devices, health care providers and pharmaceuticals manufacturers. The gap between the required growth in healthcare infrastructure and care providing staff in India cannot be addressed through an incremental and linear approach . Latest advancement in technology like artificial intelligence, internet of things (IOT) and blockchain can enable the healthcare industry to adopt disruptive led service and business models, scale up for access and affordability and take the winning leap to make India a global health care hub. Primarily, these technologies are artificial intelligence, internet of things and building information modeling However, switching to such technologies is not easy for every health care institution particularly in developing countries such as India. Adoption of such advance technologies faces many challenges. Present research focuses on such barriers and further discusses the interrelationship amongst these barriers using ISM methodology .
\end{abstract}

\section{Keywords}

Health care industry, ISM methodology, Building Information Modeling (BIM), Internet of things (IoT), Block chain technology

\section{INTRODUCTION}

Health care is one of the fastest growing sector in India and Technology is the best way to achieve the vision of a connected health care ecosystem. Medical devices in hospitals/clinics, mobile care applications, wearables and sensors are all different forms of technology that are transforming this ecosystem [1]. This enable caregivers to perform their roles more easily by automating the logging-on mechanism, automating real-time updates of patient vitals and providing insights into early detection of diseases. Technology adoption in health care in India is at nascent stage . Technologies help to collect, store and share critical patient data. By adding an analytics layer to this, caregivers can provide a much better analysis of the condition and recommendations to the patient.

One of the first step towards this transformation is to use technology as the lever to break silos and for tight coupling of data with providers and care givers and to encourage and enable an ecosystem of free data flow and interchange. Other steps will include adoption of IOT enabled wearables to monitor health, artificial intelligence enabled predictive analytics to predict diseases, mobile and IT enable technology to shift from hospital based care to technology enable home care, telemedicine and advanced imaging enabled technologies to address availability of specialists doctors in remote and rural areas. Sharing of health data will bring privacy concerns which needs to be addressed through greater focus and investment in cyber security.

Fortunately technologies have evolved and converged significantly over the last decade and shown promise to address these gaps in the Indian healthcare infrastructure . Rapid developments in mobile technologies , cloud computing digital imaging, machine learning and 3D printing have paved the way for breakthroughs in the development and adoption of healthcare technologies - from telemedicine to nanotechnology, lab grown 3D organs to internet of medical things and electronic health records to artificial intelligence . Technology enabled healthcare can provide cost effective solutions and scalable solutions to India's health care problems and make it a global health care destination. Digitally enhanced health care , specialty operating models and disruptive technology led health care services are already making strides in patient satisfaction and provide revenue in western economies.

Three common technologies used by health care facilities are :

1. Building Information Modeling (BIM) [2-3] : BIM is a perfect fit for healthcare facilities or buildings because of the complex nature of these buildings and the repetitiveness of the activity within the building. These buildings have facilities for complete patient care as well as holistic healing. By modelling healthcare projects, the early adopters of BIM are seeing reduced project costs, shortened schedules, and increased project quality. The biggest gains are the result of improved coordination of ultra-complex building systems and the ability for real-time visualization with Building Team members and clients .

2. Artificial intelligence : Artificial Intelligence (AI)[4-6] innovation has already demonstrated significant promise in healthcare by reducing costs to providers and improving quality and access to patients. Accenture predicts the healthcare AI market to be worth $\$ 6.6$ billion by 2021 and experience a $40 \%$ CAGR . By the same token, the survey results of 200 healthcare decision makers conducted by Intel and Convergys Analytics indicated that $54 \%$ expect to see the widespread adoption of AI within the next 5 years.

Internet of medical things : Internet of Medical Things (IoMT) [2,3,7]refers to the worldwide network of interconnected medical devices and applications. The healthcare sector stands to gain a lot from this technology as 
IoMT has found applications in clinical as well as nonclinical scenarios. On the clinical side, IoMT is being used to monitor a patient's vitals (temperature, blood oxygen saturation, blood pressure, respiration, ECG/EEG/EMG, etc.) and raise timely alarms. It is also being used for continuous monitoring of vitals and assisting physicians with intuitive dashboards instead of them manually operating ICU devices/patient monitors to check for recent spikes in vitals or relying on information noted manually by nurses at regular intervals. IoMT solutions available in the market are mostly proprietary targeted solutions that are developed to tailor a particular client's requirement, with an emphasis on reducing the cost and not with an intent of sharing information with external players in the future.

Primary uses of these technologies include health care delivery includes breast cancer screening ; preliminary symptoms based diagnosis, cancer treatment options . Amongst operational excellence, it includes ICU vitals monitoring and transmitting data from legacy biomedical devices . Amongst receiving customer experience, it includes patient appointment scheduling and indoor navigation [1] .

Objectives of this paper therefore is to recognize various barriers associated with the adoption of various technologies used by health care facilities particularly in developing countries such as India. Paper is organized as follows: section 2 presents the literature review. Section 3 presents the ISM methodology . Section 4 provides the hierarchical arrangements of technological barriers as per ISM methodology through a diagraph. Finally managerial implications have been provided in section 5 .

\section{LITERATURE REVIEW ON VARIOUS BARRIERS TO THESE TECHNOLOGIES}

Various barriers to technologies have been mentioned in the table below [1-13].

\section{Table 1: Various technological barriers}

\begin{tabular}{|c|c|}
\hline S.No. & Barrier and its explanation \\
\hline 1. & $\begin{array}{c}\text { Absence of a health care regulatory body (AHCB): } \\
\text { Currently, there is no healthcare regulatory body in } \\
\text { India. Data privacy and security is a concern in the } \\
\text { absence of the HDPSA, which is still being drafted } \\
\text { by the Union Health Ministry. Further, the } \\
\text { effectiveness of the Medical Devices Rules, 2017, is } \\
\text { yet to be seen. }\end{array}$ \\
\hline 2. & $\begin{array}{c}\text { Unaffordability (UA) : Further, the cost of collecting } \\
\text { and testing this data (e.g. collecting genome data) is } \\
\text { enormous. }\end{array}$ \\
\hline 3. & $\begin{array}{c}\text { Privacy and security of data (PSD) : Many medical } \\
\text { devices in the healthcare ecosystem run on public } \\
\text { networks and there are no regulations for ensuring } \\
\text { secure management of such data. There is concern } \\
\text { and confusion regarding legal aspects tracking patient } \\
\text { location. }\end{array}$ \\
\hline 4. & $\begin{array}{c}\text { Unavailability of relevant data (URD): Sharing of } \\
\text { clinical data and discussions among various } \\
\text { stakeholders should be promoted in order to } \\
\text { collaboratively draw insights from the captured data. }\end{array}$ \\
\hline 5. & $\begin{array}{c}\text { Interoperability (IO) : IoMT solutions available in the } \\
\text { market are mostly proprietary targeted solutions that }\end{array}$ \\
\hline
\end{tabular}

\begin{tabular}{|c|c|}
\hline & $\begin{array}{l}\text { are developed to tailor a particular client's } \\
\text { requirement, with an emphasis on reducing the cost } \\
\text { and not with an intent of sharing information with } \\
\text { external players in the future. Therefore, most of the } \\
\text { current implementations use different protocols } \\
\text { which result in the lack of standardisation of } \\
\text { communication protocols. This leads to } \\
\text { interoperability issues between various IoMT } \\
\text { devices/ solutions available in the market. }\end{array}$ \\
\hline 6. & Lack of appropriate infrastructure (LAI) \\
\hline 7. & Lack of education and knowledge (LEK) \\
\hline 8. & $\begin{array}{c}\text { Absence or minimum involvement and help from } \\
\text { government / policies towards high involvement of } \\
\text { technology ( AGI) }\end{array}$ \\
\hline 9. & $\begin{array}{l}\text { Difficulty in enforcing legislation and acceptance by } \\
\text { community (DEL/ absence of stronger regulations }\end{array}$ \\
\hline 10. & $\begin{array}{l}\text { Lack of appropriate and trained workforce (LTW) to } \\
\text { maintain new health technology }\end{array}$ \\
\hline 11. & $\begin{array}{c}\text { Lack of motivation and interest (LMI) on part of } \\
\text { stakeholders and less motivation and anxiety to adopt } \\
\text { the technologies }\end{array}$ \\
\hline 12. & $\begin{array}{c}\text { Organizational barriers which includes absence of } \\
\text { documented studies (ADS) }\end{array}$ \\
\hline 13. & $\begin{array}{l}\text { Financial and time constraint (FTC) in terms of cost } \\
\text { and hours to be imparted on training }\end{array}$ \\
\hline 14. & $\begin{array}{l}\text { Lack of incentives (LoI) to the deserving physicians } \\
\text { as part of motivation: most of the leaders agreed that } \\
\text { physicians spearheading the adoption of emerging } \\
\text { technologies need to be incentivised appropriately }\end{array}$ \\
\hline
\end{tabular}

Thus the 14 important barriers mentioned above have been studied further to see the inter-relationships through ISM methodology .

\section{INTERPRETIVE STRUCTURAL MODELLING METHODOLOGY}

1. Identification of elements, which are relevant to the decision maker's problems and issues.

2. Establishing the contextual relationship between elements and with respect to which pairs of elements will be examine.

3. 3. A structural self-interaction matrix (SSIM) may be developed between two variables i.e. $i$ and $j$. It establishes the "Lead to" relationship between criteria. Four symbols viz. $\mathrm{V}, \mathrm{A}, \mathrm{X} \& \mathrm{O}$ are used for establishing the relationships.

4. SSIM may be further used to develop an initial reachability matrix which has all values in binary form. Rule of transitivity is usually checked at this stage. After removing the transitivity, final reachability matrix will form.

5. Afterwards, the reachability set and antecedent set for each criterion and for each element can be obtained from the final reachability matrix.

6. After that a level partition matrix can be obtained based on establishing the precedence relationships and arranging the elements in a topological order. 
7. A Mic-Mac analysis can be performed which categorize the variables as per the driving and dependence power in to autonomous, dependent, driver and linkage category.

8. Finally a diagraph can be obtained.

\section{DEVELOPMENT OF ISM MODEL}

Table 2 : Iteration I

\begin{tabular}{|c|c|c|c|c|}
\hline S.No. & $\begin{array}{c}\text { Reachability } \\
\text { set }\end{array}$ & $\begin{array}{c}\text { Antecedent } \\
\text { set }\end{array}$ & $\begin{array}{c}\text { Intersectio } \\
\text { n set }\end{array}$ & $\begin{array}{c}\text { Iterati } \\
\text { on/ } \\
\text { Levels }\end{array}$ \\
\hline 1 & 4 & $\begin{array}{c}1,2,3,4,5,6,7,8 \\
9,10,11,12,13, \\
14\end{array}$ & 4 & \\
\hline 2 & 4,5 & $\begin{array}{c}1,2,3,5,6,7,8,9 \\
10,11,12,13, \\
14\end{array}$ & 5 & \\
\hline 3 & $3,4,5$ & $\begin{array}{c}1,2,3,6,7,8,9 \\
10,11,12,13 \\
14\end{array}$ & 3 & \\
\hline 4 & $3,4,5,12$ & $\begin{array}{c}1,2,6,7,8,9,10 \\
11,12,13,14\end{array}$ & 12 & \\
\hline 5 & $\begin{array}{c}3,4,5,6,7,10 \\
11,12\end{array}$ & $\begin{array}{c}1,2,6,7,8,9,10 \\
11,13,14\end{array}$ & $6,7,10,11$ & \\
\hline 6 & $\begin{array}{c}3,4,5,6,7,10 \\
11,12,14\end{array}$ & $\begin{array}{c}1,2,6,7,8,9,11 \\
13,14\end{array}$ & $6,7,11,14$ & \\
\hline 7 & $\begin{array}{c}3,4,5,6,7,8,10 \\
11,12,14\end{array}$ & $\begin{array}{c}1,2,6,7,8,13 \\
14\end{array}$ & $6,7,8,14$ & $\mathbf{I}$ \\
\hline 8 & $\begin{array}{c}2,3,4,5,6,7,8,1 \\
0,11,12,14\end{array}$ & $2,9,13$ & 2 & \\
\hline 9 & $\begin{array}{c}2,3,4,5,6,7,8,9 \\
10,11,12,14\end{array}$ & 9,13 & 9 & \\
\hline 10 & $\begin{array}{l}2,3,4,5,6,7,8,9 \\
10,11,12,13,14\end{array}$ & 13 & 13 & \\
\hline 11 & $\begin{array}{c}1,2,3,4,5,6,7,8 \\
9,10,11,12,13 \\
14\end{array}$ & 1 & 1 & \\
\hline
\end{tabular}

In this section, ISM model is developed for studying the interrelationships amongst various barriers associated with the adoption of various technologies used by health care facilities in developing countries. Fourteen important criteria are Absence of a health care regulatory body (AHCB); Unaffordability (UA); absence of privacy and security of health care related data (APSD); Unavailability of relevant data (URD) ; Interoperability (IO) ; Lack of appropriate infrastructure (LAI) ; Lack of education and knowledge (LEK) ; Absence of government involvement towards technology advancements( AGI) ; Difficulty in enforcing legislation and acceptance by community (DEL) ; Lack of appropriate and trained workforce (LTW) to maintain new health technology ; Lack of motivation and interest (LMI) ; absence of documented studies (ADS) ; Financial and time constraint (FTC) ; Lack of incentives (LoI) .

\subsection{Construction of Structural Self - Interaction Matrix (SSIM)}

This matrix gives the pair-wise relationship between two variables i.e. $i$ and $j$ based on VAXO. SSIM has been presented below in Fig 1.

\section{Explanation:}

Security issues lead to interoperability issues . lack of appropriate infrastructure may lead to lack of education and vice versa . lack of infrastructure may lead to lack of trained workforce . Lack of infrastructure may also lead to lack of motivation. Absence of government involvement may lead to absence of infrastructure and vice versa as well as lack of education. Lack of government involvement may also lead to difficulty in enforcing legislation. Lack of infrastructure may lead to lack of incentives. Difficulty in enforcing legislation may lead to difficulty in getting trained workforce.

\section{2 ikConstruction of Initial Reachability Matrix and final reachability matrix}

The SSIM has been converted in to a binary matrix called the initial reachability matrix shown in fig. 2 by substituting V, A, $\mathrm{X}, \mathrm{O}$ by 1 or 0 as per the case. After incorporating the transitivity, the final reachability matrix is shown below in the Fig 3.

\subsection{Level Partition}

From the final reachability matrix, reachability and final antecedent set for each factor are found. The elements for which the reachability and intersection sets are same are the top-level element in the ISM hierarchy. After the identification of top level element, it is separated out from the other elements and the process continues for next level of elements. Reachability set, antecedent set, intersection set along with different level for elements have been shown below in table 2 to table 12 . 
Fig 1: Structural Self- Interaction Matrix

\begin{tabular}{|c|c|c|c|c|c|c|c|c|c|c|c|c|c|c|c|}
\hline Barrier & & 1 & 2 & 3 & 4 & 5 & 6 & 7 & 8 & 9 & 10 & 11 & 12 & 13 & 14 \\
\hline & & $\begin{array}{c}\mathrm{AHC} \\
\mathrm{B}\end{array}$ & UA & $\begin{array}{l}\text { AP } \\
\text { SD }\end{array}$ & URD & $\mathrm{IO}$ & LAI & LEK & $\begin{array}{c}\mathrm{AG} \\
\mathrm{I}\end{array}$ & $\begin{array}{c}\mathrm{DE} \\
\mathrm{L}\end{array}$ & LTW & LMI & $\begin{array}{c}\mathrm{AD} \\
\mathrm{S}\end{array}$ & FTC & LoI \\
\hline 1. & AHCB & & $\mathrm{O}$ & $\mathrm{O}$ & $\mathrm{O}$ & $\mathrm{V}$ & V & V & $X$ & $\mathrm{~V}$ & V & V & V & $\mathrm{O}$ & $\mathrm{V}$ \\
\hline 2. & UA & & & $\mathrm{V}$ & $\mathrm{V}$ & $\mathrm{O}$ & $\mathrm{V}$ & $\mathrm{V}$ & A & $\mathrm{V}$ & $\mathrm{V}$ & $\mathrm{V}$ & $\mathrm{V}$ & A & $\mathrm{V}$ \\
\hline 3. & APSD & & & & $\mathrm{V}$ & $\mathrm{V}$ & A & A & A & $\mathrm{A}$ & A & A & A & A & A \\
\hline 4. & URD & & & & & $\mathrm{O}$ & $\mathrm{A}$ & $\mathrm{A}$ & A & $\mathrm{A}$ & A & $\bar{A}$ & $\mathrm{~A}$ & A & $\mathrm{A}$ \\
\hline 5. & $\mathrm{IO}$ & & & & & & A & $\mathrm{A}$ & A & $\mathrm{A}$ & $\mathrm{A}$ & $\mathrm{A}$ & A & A & $\mathrm{A}$ \\
\hline 6. & LAI & & & & & & & $\mathrm{X}$ & $\mathrm{X}$ & $\mathrm{A}$ & $\mathrm{V}$ & $\mathrm{V}$ & $\mathrm{V}$ & A & $\mathrm{X}$ \\
\hline 7. & LEK & & & & & & & & A & $\mathrm{A}$ & $\mathrm{X}$ & A & $\mathrm{V}$ & A & $\mathrm{A}$ \\
\hline 8. & AGI & & & & & & & & & $\mathrm{V}$ & $\mathrm{V}$ & $\mathrm{V}$ & $\mathrm{X}$ & $\mathrm{V}$ & $\mathrm{V}$ \\
\hline 9. & DEL & & & & & & & & & & V & V & V & V & $\mathrm{V}$ \\
\hline 10. & LTW & & & & & & & & & & & V & V & A & A \\
\hline 11. & LMI & & & & & & & & & & & & V & A & A \\
\hline 12. & ADS & & & & & & & & & & & & & A & $\mathrm{A}$ \\
\hline 13. & FTC & & & & & & & & & & & & & & $\mathrm{A}$ \\
\hline 14. & LoI & & & & & & & & & & & & & & \\
\hline
\end{tabular}

Fig 2: Initial reachability matrix

\begin{tabular}{|c|c|c|c|c|c|c|c|c|c|c|c|c|c|c|c|}
\hline Barrie & & 1 & 2 & 3 & 4 & 5 & 6 & 7 & 8 & 9 & 10 & 11 & 12 & 13 & 14 \\
\hline & & $\begin{array}{c}\mathrm{AHC} \\
\mathrm{B}\end{array}$ & UA & $\begin{array}{l}\text { AP } \\
\text { SD }\end{array}$ & URD & IO & LAI & LEK & $\begin{array}{c}\mathrm{AG} \\
\mathrm{I}\end{array}$ & $\begin{array}{c}\mathrm{DE} \\
\mathrm{L}\end{array}$ & LTW & LMI & $\begin{array}{c}\mathrm{AD} \\
\mathrm{S}\end{array}$ & FTC & LoI \\
\hline 1. & AHCB & 1 & 0 & 0 & 0 & 1 & 1 & 1 & 1 & 1 & 1 & 1 & 1 & 0 & 1 \\
\hline 2. & UA & 0 & 1 & 1 & 1 & 0 & 1 & 1 & 0 & 1 & 1 & 1 & 1 & 0 & 1 \\
\hline 3. & APSD & 0 & 0 & 1 & 1 & 1 & 0 & 0 & 0 & 0 & 0 & 0 & 0 & 0 & 0 \\
\hline 4. & URD & 0 & 0 & 0 & 1 & 0 & 0 & 0 & 0 & 0 & 0 & 0 & 0 & 0 & 0 \\
\hline 5. & $\mathrm{IO}$ & 0 & 0 & 0 & 0 & 1 & 0 & 0 & 0 & 0 & 0 & 0 & 0 & 0 & 0 \\
\hline 6. & LAI & 0 & 0 & 1 & 1 & 1 & 1 & 1 & 1 & 0 & 1 & 1 & 1 & 0 & 1 \\
\hline 7. & LEK & 0 & 0 & 1 & 1 & 1 & 1 & 1 & 0 & 0 & 1 & 0 & 1 & 0 & 0 \\
\hline 8. & AGI & 1 & 1 & 1 & 1 & 1 & 1 & 1 & 1 & 1 & 1 & 1 & 1 & 1 & 1 \\
\hline 9. & DEL & 0 & 1 & 1 & 1 & 1 & 1 & 1 & 0 & 1 & 1 & 1 & 1 & 1 & 1 \\
\hline 10. & LTW & 0 & 1 & 1 & 1 & 1 & 0 & 1 & 0 & 0 & 1 & 1 & 1 & 0 & 0 \\
\hline 11. & LMI & 1 & 1 & 1 & 1 & 1 & 0 & 1 & 0 & 0 & 0 & 1 & 1 & 0 & 0 \\
\hline 12. & ADS & 0 & 0 & 1 & 1 & 1 & 0 & 0 & 1 & 0 & 0 & 0 & 1 & 0 & 0 \\
\hline 13. & FTC & 0 & 1 & 1 & 1 & 1 & 1 & 1 & 0 & 0 & 1 & 1 & 1 & 1 & 0 \\
\hline 14. & LoI & 0 & 0 & 1 & 1 & 1 & 1 & 1 & 0 & 0 & 1 & 1 & 1 & 1 & 1 \\
\hline
\end{tabular}


Fig 3 : Final reachability matrix

\begin{tabular}{|c|c|c|c|c|c|c|c|c|c|c|c|c|c|c|c|c|}
\hline Barrie & & 1 & 2 & 3 & 4 & 5 & 6 & 7 & 8 & 9 & 10 & 11 & 12 & 13 & 14 & D.P \\
\hline & & $\begin{array}{l}\mathrm{AH} \\
\mathrm{CB}\end{array}$ & UA & $\begin{array}{l}\mathrm{AP} \\
\mathrm{SD}\end{array}$ & $\begin{array}{l}\text { UR } \\
\text { D }\end{array}$ & $\mathrm{IO}$ & LAI & LEK & $\begin{array}{l}\text { A } \\
\text { GI }\end{array}$ & $\begin{array}{l}\mathrm{DE} \\
\mathrm{L}\end{array}$ & $\begin{array}{l}\mathrm{LT} \\
\mathrm{W}\end{array}$ & $\begin{array}{l}\text { LM } \\
\text { I }\end{array}$ & $\begin{array}{l}\mathrm{AD} \\
\mathrm{S}\end{array}$ & $\begin{array}{l}\text { FT } \\
\text { C }\end{array}$ & LoI & \\
\hline 1. & AHCB & 1 & 0 & 1 & 1 & 1 & 1 & 1 & 1 & 1 & 1 & 1 & 1 & 0 & 1 & 12 \\
\hline 2. & UA & 0 & 1 & 1 & 1 & 1 & 1 & 1 & 1 & 1 & 1 & 1 & 1 & 0 & 1 & 12 \\
\hline 3. & APSD & 0 & 0 & 1 & 1 & 1 & 0 & 0 & 0 & 0 & 0 & 0 & 0 & 0 & 0 & 3 \\
\hline 4. & URD & 0 & 0 & 0 & 1 & 0 & 0 & 0 & 0 & 0 & 0 & 0 & 0 & 0 & 0 & 1 \\
\hline 5. & IO & 0 & 0 & 0 & 1 & 1 & 0 & 0 & 0 & 0 & 0 & 0 & 0 & 0 & 0 & 2 \\
\hline 6. & LAI & 0 & 0 & 1 & 1 & 1 & 1 & 1 & 1 & 1 & 1 & 1 & 1 & 1 & 1 & 12 \\
\hline 7. & LEK & 0 & 0 & 1 & 1 & 1 & 1 & 1 & 1 & 0 & 1 & 1 & 1 & 0 & 1 & 10 \\
\hline 8. & AGI & 1 & 1 & 1 & 1 & 1 & 1 & 1 & 1 & 1 & 1 & 1 & 1 & 1 & 1 & 14 \\
\hline 9. & DEL & 0 & 1 & 1 & 1 & 1 & 1 & 1 & 0 & 1 & 1 & 1 & 1 & 1 & 1 & 12 \\
\hline 10. & LTW & 0 & 1 & 1 & 1 & 1 & 1 & 1 & 0 & 1 & 1 & 1 & 1 & 0 & 0 & 10 \\
\hline 11. & LMI & 0 & 1 & 1 & 1 & 1 & 1 & 1 & 0 & 0 & 1 & 1 & 1 & 0 & 1 & 10 \\
\hline 12. & ADS & 0 & 0 & 1 & 1 & 1 & 0 & 0 & 1 & 0 & 0 & 0 & 1 & 0 & 0 & 5 \\
\hline 13. & FTC & 0 & 1 & 1 & 1 & 1 & 1 & 1 & 1 & 0 & 1 & 1 & 1 & 1 & 1 & 12 \\
\hline \multirow[t]{2}{*}{14.} & LoI & 0 & 1 & 1 & 1 & 1 & 1 & 1 & 1 & 0 & 1 & 1 & 1 & 1 & 1 & 12 \\
\hline & De.P & 2 & 7 & 12 & 14 & 13 & 10 & 10 & 8 & 6 & 10 & 10 & 11 & 5 & 9 & \\
\hline
\end{tabular}

D.P : Driving power ; De.P : dependence power

Table 3 : Iteration II

\begin{tabular}{|c|c|c|c|c|}
\hline S.No. & Reachability set & Antecedent set & Intersection set & Iteration \\
\hline 2 & 5 & $1,2,3,5,6,7,8,9,10,11,12,13,14$ & 5 & \multirow{10}{*}{ II } \\
\hline 3 & 3,5 & $1,2,3,6,7,8,9,10,11,12,13,14$ & 3 & \\
\hline 4 & $3,5,12$ & $\begin{array}{c}1,2,6,7,8,9 \\
10,11,12,13 \\
14\end{array}$ & 12 & \\
\hline 5 & $\begin{array}{c}3,5,6,7,10,11 \\
12\end{array}$ & $\begin{array}{c}1,2,6,7,8,9 \\
10,11,13,14\end{array}$ & $6,7,10,11$ & \\
\hline 6 & $\begin{array}{c}3,5,6,7,10,11 \\
12,14\end{array}$ & $\begin{array}{c}1,2,6,7,8,9 \\
11,13,14\end{array}$ & $6,7,11,14$ & \\
\hline 7 & $3,5,6,7,8,10,11,12,14$ & $1,2,6,7,8,13,14$ & $6,7,8,14$ & \\
\hline 8 & $2,3,5,6,7,8,10,11,12,14$ & $2,9,13$ & 2 & \\
\hline 9 & $\begin{array}{c}2,3,5,6,7,8,9 \\
10,11,12,14\end{array}$ & 9,13 & 9 & \\
\hline 10 & $\begin{array}{c}2,3,5,6,7,8,9 \\
10,11,12,13,14\end{array}$ & 13 & 13 & \\
\hline 11 & $1,2,3,5,6,7,8,9,10,11,12,13,14$ & 1 & 1 & \\
\hline
\end{tabular}


Table 4 : Iteration III

\begin{tabular}{|c|c|c|c|c|}
\hline $\begin{array}{l}\text { Sr. } \\
\text { No. }\end{array}$ & $\begin{array}{c}\text { Reachab } \\
\text { ility set }\end{array}$ & Antecedent set & $\begin{array}{c}\text { Intersectio } \\
\text { n set }\end{array}$ & $\begin{array}{l}\text { Itera } \\
\text { tion }\end{array}$ \\
\hline 3 & 3 & $\begin{array}{c}1,2,3,6,7,8,9,10 \\
11,12,13,14\end{array}$ & 3 & \multirow{9}{*}{ III } \\
\hline 4 & 3,12 & $\begin{array}{c}1,2,6,7,8,9,10,11,12,13, \\
14\end{array}$ & 12 & \\
\hline 5 & $\begin{array}{c}3,6,7,10, \\
11,12\end{array}$ & $1,2,6,7,8,9,10,11,13,14$ & $6,7,10,11$ & \\
\hline 6 & $\begin{array}{l}3,6,7,10 \\
11,12,14\end{array}$ & $1,2,6,7,8,9,11,13,14$ & $6,7,11,14$ & \\
\hline 7 & $\begin{array}{c}3,6,7,8,1 \\
0,11,12 \\
14\end{array}$ & $1,2,6,7,8,13,14$ & $6,7,8,14$ & \\
\hline 8 & $\begin{array}{c}2,3,6,7,8 \\
, 10,11,1 \\
2,14\end{array}$ & $2,9,13$ & 2 & \\
\hline 9 & $\begin{array}{c}2,3,6,7,8 \\
, 9 \\
10,11,12 \\
, 14\end{array}$ & 9,13 & 9 & \\
\hline 10 & $\begin{array}{c}2,3,6,7,8 \\
, 9, \\
10,11,12 \\
, 13,14\end{array}$ & 13 & 13 & \\
\hline 11 & $\begin{array}{c}1,2,3,6,7 \\
8,9,10,1 \\
1,12,13 \\
14\end{array}$ & 1 & 1 & \\
\hline
\end{tabular}

Table 6 : Iteration IV

\begin{tabular}{|c|c|c|c|c|}
\hline $\begin{array}{l}\text { S. } \\
\text { No. }\end{array}$ & $\begin{array}{c}\text { Reachabilit } \\
\text { y set }\end{array}$ & $\begin{array}{c}\text { Antecedent } \\
\text { set }\end{array}$ & $\begin{array}{c}\text { Intersection } \\
\text { set }\end{array}$ & $\begin{array}{l}\text { Iterat } \\
\text { ion/ } \\
\text { Level } \\
\text { s }\end{array}$ \\
\hline 4 & 12 & $\begin{array}{c}1,2,6,7,8,9,1 \\
0,11,12,13 \\
14\end{array}$ & 12 & \multirow{8}{*}{ IV } \\
\hline 5 & $6,7,10,11,12$ & $\begin{array}{c}1,2,6,7,8,9,1 \\
0,11,13,14\end{array}$ & $6,7,10,11$ & \\
\hline 6 & $\begin{array}{c}6,7,10,11,12 \\
14\end{array}$ & $\begin{array}{c}1,2,6,7,8,9,1 \\
1,13,14\end{array}$ & $6,7,11,14$ & \\
\hline 7 & $\begin{array}{c}6,7,8,10,11,1 \\
2,14\end{array}$ & $\begin{array}{c}1,2,6,7,8,13, \\
14\end{array}$ & $6,7,8,14$ & \\
\hline 8 & $\begin{array}{c}2,6,7,8,10,11 \\
, 12,14\end{array}$ & $2,9,13$ & 2 & \\
\hline 9 & $\begin{array}{c}2,6,7,8,9,10 \\
11,12,14\end{array}$ & 9,13 & 9 & \\
\hline 10 & $\begin{array}{c}2,6,7,8,9,10 \\
11,12,13,14\end{array}$ & 13 & 13 & \\
\hline 11 & $\begin{array}{c}1,2,6,7,8,9,1 \\
0,11,12,13 \\
14\end{array}$ & 1 & 1 & \\
\hline
\end{tabular}

Table 7 : Iteration V

\begin{tabular}{|c|c|c|c|c|}
\hline $\begin{array}{l}\text { Sr. } \\
\text { No. }\end{array}$ & $\begin{array}{c}\text { Reachability } \\
\text { set }\end{array}$ & $\begin{array}{c}\text { Antecedent } \\
\text { set }\end{array}$ & $\begin{array}{c}\text { Intersection } \\
\text { set }\end{array}$ & $\begin{array}{l}\text { Itera } \\
\text { tion }\end{array}$ \\
\hline 5 & $6,7,10,11$ & $\begin{array}{c}1,2,6,7,8,9,10,1 \\
1,13,14\end{array}$ & $6,7,10,11$ & \\
\hline 6 & $6,7,10,11,14$ & $\begin{array}{c}1,2,6,7,8,9,11,1 \\
3,14\end{array}$ & $6,7,11,14$ & \\
\hline 7 & $\begin{array}{c}6,7,8,10,11,1 \\
4\end{array}$ & $\begin{array}{c}1,2,6,7,8,13 \\
14\end{array}$ & $6,7,8,14$ & \\
\hline 8 & $\begin{array}{c}2,6,7,8,10,11 \\
, 14\end{array}$ & $2,9,13$ & 2 & \\
\hline 9 & $\begin{array}{c}2,6,7,8,9,10 \\
11,14\end{array}$ & 9,13 & 9 & \\
\hline 10 & $\begin{array}{c}2,6,7,8,9,10 \\
11,13,14\end{array}$ & 13 & 13 & \\
\hline 11 & $\begin{array}{c}1,2,6,7,8,9, \\
10,11,13,14\end{array}$ & 1 & 1 & $\mathbf{V}$ \\
\hline
\end{tabular}

Table 8 : Iteration VI

\begin{tabular}{|c|c|c|c|c|}
\hline $\begin{array}{c}\text { Sr. } \\
\text { No. }\end{array}$ & $\begin{array}{c}\text { Reachability } \\
\text { set }\end{array}$ & Antecedent set & $\begin{array}{c}\text { Intersection } \\
\text { set }\end{array}$ & $\begin{array}{c}\text { Itera } \\
\text { tion }\end{array}$ \\
\hline 6 & 14 & $1,2,8,9,13,14$ & $6,7,14$ & \\
\cline { 1 - 4 } & $\mathbf{8 , 1 4}$ & $1,2,6,7,8,13,14$ & $6,7,8,14$ & \\
\cline { 1 - 4 } 8 & $2,8,14$ & $2,9,13$ & 2 & \\
\hline 9 & $2,8,9,14$ & 9,13 & 9 & \multirow{2}{*}{ VI } \\
\hline 10 & $2,8,9,13,14$ & 13 & 13 & \\
\hline 11 & $1,2,8,9,13,14$ & 1 & & \\
\end{tabular}

Table 9 : Iteration VII

\begin{tabular}{|c|c|c|c|c|}
\hline $\begin{array}{c}\text { Sr. } \\
\text { No. }\end{array}$ & $\begin{array}{c}\text { Reachability } \\
\text { set }\end{array}$ & $\begin{array}{c}\text { Antecedent } \\
\text { set }\end{array}$ & $\begin{array}{c}\text { Intersection } \\
\text { set }\end{array}$ & $\begin{array}{c}\text { Itera } \\
\text { tion }\end{array}$ \\
\hline 8 & $\mathbf{2}$ & $2,9,13$ & 2 & \\
& 2,9 & 9,13 & 9 & \multirow{2}{*}{ VII } \\
\hline 10 & $2,9,13$ & 13 & 13 & \\
\hline 11 & $1,2,9,13$ & 1 & 1 & \\
\hline
\end{tabular}

Table 10 : Iteration VIII

\begin{tabular}{|c|c|c|c|c|}
\hline $\begin{array}{c}\text { Sr. } \\
\text { No. }\end{array}$ & $\begin{array}{c}\text { Reachability } \\
\text { set }\end{array}$ & $\begin{array}{c}\text { Antecedent } \\
\text { set }\end{array}$ & $\begin{array}{c}\text { Intersection } \\
\text { set }\end{array}$ & $\begin{array}{c}\text { Itera } \\
\text { tion }\end{array}$ \\
\hline 9 & $\mathbf{9}$ & 9,13 & 9 & VIII \\
\hline 10 & 9,13 & 13 & 13 & \\
\hline 11 & $1,9,13$ & 1 & 1 & \\
\hline
\end{tabular}


Table 11 : Iteration IX

\begin{tabular}{|c|c|c|c|c|}
\hline $\begin{array}{c}\text { Sr. } \\
\text { No. }\end{array}$ & $\begin{array}{c}\text { Reachability } \\
\text { set }\end{array}$ & $\begin{array}{c}\text { Antecedent } \\
\text { set }\end{array}$ & $\begin{array}{c}\text { Intersection } \\
\text { set }\end{array}$ & $\begin{array}{c}\text { Itera } \\
\text { tion }\end{array}$ \\
\hline 10 & $\mathbf{1 3}$ & 13 & 13 & IX \\
\hline 11 & 1,13 & 1 & 1 & \\
\hline
\end{tabular}

Table 12 : Iteration $\mathrm{X}$

\begin{tabular}{|c|c|c|c|c|}
\hline $\begin{array}{c}\text { Sr. } \\
\text { No. }\end{array}$ & $\begin{array}{c}\text { Reachability } \\
\text { set }\end{array}$ & $\begin{array}{c}\text { Antecedent } \\
\text { set }\end{array}$ & $\begin{array}{c}\text { Intersection } \\
\text { set }\end{array}$ & $\begin{array}{c}\text { Itera } \\
\text { tion }\end{array}$ \\
\hline 10 & $\mathbf{1}$ & 1 & 1 & $\mathbf{X}$ \\
\hline
\end{tabular}

\subsection{Classification of factors}

The critical success factors described earlier are classified in to four clusters viz. autonomous factor, dependent factors, linkage factors and independent factors (mentioned in Table XII below). Fig. 4 below shows the driving power and dominance diagram

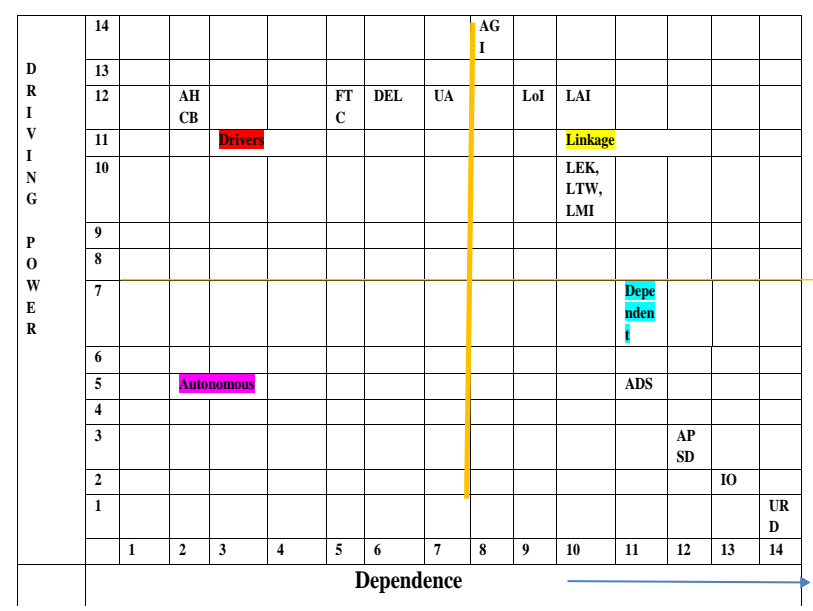

Fig . 4: Driving power and dependence diagram

\subsection{ISM model}

An ISM model is developed ( as shown in fig. 5 below ) after arranging the elements as per their interaction or dependence relationships.

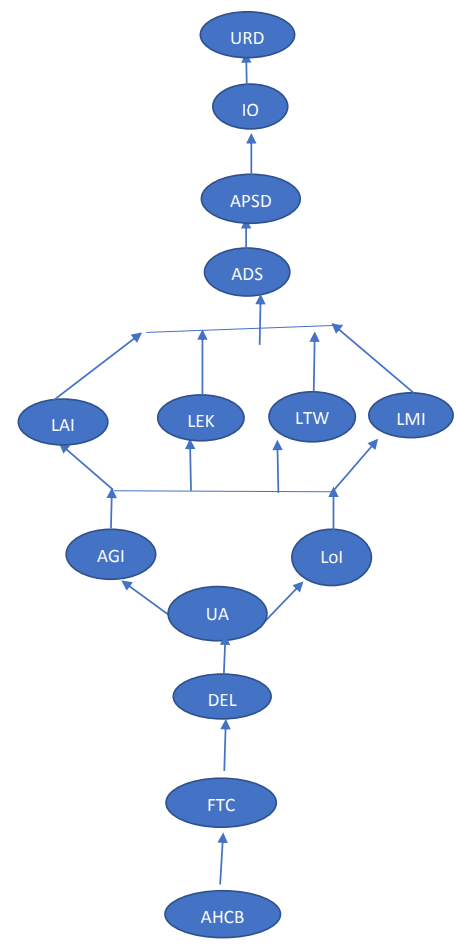

Fig 5: ISM diagraph

\section{CONCLUSIONS \& MANAGERIAL IMPLICATIONS}

1. The greatest benefit managers derive from these technologies is that these techniques are known for converting visualization to reality. It brings the team effort as with BIM at their disposal, architects, contractors and civil engineers can come together and communicate the appeal, aesthetics, constructability and every other detail of the project to ensure that the design translates into reality exactly as visualized.

2. Another advantage of BIM deals with reducing wastage of time , talent, resources and need for rework. These activities are quite necessary and helpful for healthcare facility projects as it delivers high value to clients via efficient design and construction .

3. Visual, design and construction (VDC) efficacies of BIM helps to communicate the most complex processes of the construction. They are also known for clash detection and risk mitigation .

4. BIM project managers ensure that everything right from gauging the required material quantity, to fabrication, erection and construction of the building can be well planned and coordinated using BIM.

5. Rework, clashes and risks are greatly reduced and contractors can also manage spatial requirements on construction site capably with BIM. The construction schedules can be well coordinated, allowing projects to complete on time.

6. For a hospital building, it is important to consider spatial requirements and equipment layouts as there are severa types of diagnostic, testing and treatment requirements to consider. The building information models are loaded 
with visualizations for placement of all these equipment, which in turn benefits the facility management phase.

7. Building information modeling allows an effective blending of design and aesthetics thereby making these facilities stylishly designed to suit the needs of its patients .

8. The techniques such as artificial intelligence amplify the capabilities of the staff .

9. BIM models are used in health care facilities to create walkthroughs. These walkthroughs could be of the trauma rooms or operation theatre. Multiple renderings could be produced to demonstrate the spatial relationships of the different configurations, helping the client to understand the impact of their decisions on the room, corridor, and exterior.

10. Despite the enormous costs related to the collection and testing of clinical data such as genome data, some startups have started collecting India specific data and plan on extrapolating it for it to become relevant for AI.

11. Artificial intelligence technology is built on gaining trust by ensuring transparency in all aspects of the technology. Managers are usually advised to consider using AI to automate simple tasks before deploying it for more complex ones.

12. Usually these technologies causes a shift in workforce skills particularly higher cognitive skills and social and emotional skills. This causes the feeling of insecurity amongst the workers about their job. This increases the responsibility on part of manager .

13. ROI metrics can be refined to include increasing revenue and reducing costs. Integrating AI insights effectively into workflows by enabling the seamless exchange of data from one source to another is another managers task associated with these technologies

\section{ACKNOWLEDGEMENT}

Authors are thankful to Prof. S.P Singh, DMS , IIT Delhi for imparting the knowledge on ISM methodology and Computer Science Department (CSD), BITS Pilani, Pilani for disseminating knowledge on Building information Modeling (BIM) which has substantially helped authors in preparing this manuscript

\section{REFERENCES}

[1] https://www.datapine.com/kpi-examples-and- PWC and CII (2017), " how mhealth can revolutionize the Indian healthcare industry", https://www.pwc.in/assets/pdfs/publications/2017/howmhealth-an revolutionize the Indian health care industry.
[2] Reimagining the possible in the Indian healthcare ecosystem with emerging technologies, the Bengal chamber report , 2014https://www.bdcnetwork.com/bimhealthcare

[3] The medical futurist Institute (2018) . Six challenges to tackle before artificial intelligence redesigns healthcare http://medicalfuturist.com/sixchallengestotacklebeforeart ificialintelligenceredesignshealthcare

[4] Huynh , N. 2018. 4 barriers to adopting artificial intelligence and how to overcome them , https://healthcare weekly.com/adopting artificial intelligence in healthcare , 2018.

[5] Matshazi , N. 2019. How amazon is using AI for its health initiatives .

[6] Growth Enabler (2017). Market pulse report, internet of things (IOT) . Discover key trends and insights on disruptive technologies in IOT innovations. https://growthenabler.com/flipbook/pdf/IOT\%20report.p df

[7] Oak, R.M. 2007. A review on barriers to implementing health informatics in developing countries. Journal of Health Informatics in developing countries, 1(1).

[8] Sluijs MB, Veeken H, Overbeke AJ. 2006. Deficient information in developing countries: Internet alone is no solution, Ned Tijdschr Geneeskd. 150(24):1351-4, Dutch, PMID: 16808368,

[9] Chinnock P. 2004. Global review on access to health information in developing countries. The role of systematic reviews, Cochrane Collaboration, http://www.hi-urope.co.uk/files/2004/9962.htm

[10] Anwar , F. , Shamim , A. 2011. Barriers in Adoption of Health Information Technology in Developing Societies ,International Journal of Advanced Computer Science and Applications, 2(8).

[11] Bianchard ,A. Prior , F. Barton , J. Dawson, T. 2010. Barriers in new health and social care technology implementation in the united kingdom , a case study ,1, pp. 62-67. International conference on developments in e-systems engineering .

[12] Azubuike M. C., Ehiri , J.E. 1999. Health information systems in developing countries: benefits, problems, and prospects, The Journal of the Royal Society for the Promotion of Health, 119(3), pp 180-184 , DOI: $10.1177 / 146642409911900309$.

[13] Warfield, J.N. 1974. Developing interconnection matrices in structural modeling. IEEE Transactions on Systems, Man, and Cybernetics, (1), 81-87. 